\title{
Correction to: Paediatric Kawasaki Disease
}

Hong Wang

Correction to: H. Wang (ed.), Paediatric Kawasaki Disease, https://doi.org/10.1007/978-981-15-0038-1

The original version of this book was inadvertently published before updating author corrections. All the author corrections have been updated in this revised version.

The updated online version of this book can be found at https://doi.org/10.1007/978-981-15-0038-1 